\title{
$=$ \\ MODELOS DE FINANCIACIÓN DE UNA RENTA BÁSICA PARA ESPAÑA
}

\section{FINANCIAL MODELS FOR A BASIC INCOME IN SPAIN}

\author{
Javier Díaz Oyarzábal \\ Universidad Politécnica de Madrid (UPM) \\ javier.diaz.oyarzabal@alumnos.upm.es \\ Juan A. Gimeno Ullastres \\ Universidad de Educación a Distancia (UNED) \\ jgimeno@cee.uned.es \\ Víctor Gómez Frías \\ Universidad Politécnica de Madrid (UPM) \\ victor.gomez.frias@upm.es
}

Fecha recepción artículo: 28/06/2019 • Fecha de aprobación: 10/09/2019

\section{RESUMEN}

Para un colectivo creciente de expertos y responsables sociales, resulta razonable admitir que un modelo de renta básica, automática e incondicionada es técnicamente más eficiente que el vigente conjunto de medidas de sostenimiento de rentas. Pero aparece una duda paralizante y, para muchos invalidante: la impresión de que una propuesta como esa resulta imposible de financiar razonablemente.

En esta década, se han ofrecido en España al menos dos intentos significativos de cuantificar las necesidades para la implantación de una renta básica, acompañados de una propuesta financieramente viable para la puesta en marcha de la medida.

El primero de Arcarons, Raventós y Torrens (2013 y ss.); el segundo de Gimeno (2014 y ss.), con muchas coincidencias pero también con bastantes diferencias ${ }^{1}$. En este artículo se recordarán esos dos modelos de financiación de una renta básica en España, repasaremos las principales cuestiones e hipótesis, intentaremos actualizar las estimaciones ${ }^{2}$ y concluiremos con posibles propuestas de financiación, simplemente "continuistas" de aquellas propuestas originales. También abriremos el debate a otras alternativas.

Palabras Clave: renta básica, pobreza, renta mínima automática, garantía de ingresos.

\footnotetext{
${ }^{1}$ Los tres primeros autores han publicado una nueva propuesta con posterioridad a la realización de nuestras estimaciones. Se hará referencia a ese estudio más adelante.

${ }^{2}$ La actualización se fundamenta en el trabajo Estudio de Renta Básica Universal en España, realizado por Javier Díaz Oyarzábal (2019) en la UPM, tutelado por Víctor Gómez Frías.
} 


\section{ABSTRACT}

For a growing collective of experts and social responsible, it is reasonable to admit that a model of automatic and unconditional basic income, is technically more efficient than the current set of measures of income support. But a doubt appears, paralyzing and, for many, disabling: the impression that a proposal like that is impossible to finance reasonably. In this decade, at least two significant attempts to quantify the needs for the implementation of a basic income have been featured in Spain, together by a financially-viable proposal to set up measurement. The first one, Arcarons, Raventós and Torrens (2013 and ff.). The second one, Gimeno (2014 and ff.), with many matches but also some differences. In this article, we recall these two models of financing a basic income in Spain. We will review the main issues and assumptions, we will try to update estimations and we will conclude with possible funding proposals, essentially in continuity of those original proposals. We will also open the debate to other alternatives.

Keywords: basic income, poverty, automatic minimum income, guarantee of income

Javier Díaz Oyarzábal es graduado en Ingeniería en Tecnologías Industriales por la Universidad Politécnica de Madrid, habiendo cursado su último año de grado con una beca Erasmus, en École Nationale des Ponts et Chaussées, Francia. Actualmente continúa sus estudios en primero de Máster Habilitante en Ingeniería Industrial en la UPM, que compaginó con unas prácticas en la consultora Accenture.

Juan A. Gimeno se licenció en Ciencias Económicas y Empresariales y Derecho en la Universidad Complutense de Madrid, donde doctoró con premio extraordinario y en la que inició su carrera docente. Es Catedrático de Economía Aplicada desde 1986 en la UNED, universidad de la que fue Rector. Ha recibido premios de investigación del Instituto de Estudios Fiscales y del CES. Fue el primer director de la Revista Española del Tercer Sector y es fundador y patrono de Economistas sin Fronteras.

Víctor Gómez Frías (UPM) es profesor en la Escuela Técnica Superior de Ingenieros Industriales de la Universidad Politécnica de Madrid, donde estudió ingeniería de Caminos, Canales y Puertos. Realizó un doble diploma en la École Nationale des Ponts et Chaussées, donde fue profesor titular y subdirector académico. Es doctor en Economía de la Empresa por la École Polytechnique, y licenciado en Derecho y en Ciencias Politícas en la UNED. 


\section{DEFINICIÓN DE LOS MODELOS DE RENTA BÁSICA Y COSTE ANUAL}

La primera decisión fundamental para estimar el coste de una posible renta básica es fijar su cuantía. Conviene recordar que los dos trabajos que sirven de referencia inicial (uno de Arcarons et al. y otro de Gimeno) parten de datos del año 2010. En las actualizaciones intentaremos traer las cifras más recientes de las que disponemos.

Arcarons, Raventós y Torrens (2013 y ss.) parten de la cuantía necesaria para eliminar la pobreza, fijando consecuentemente el importe necesario en el $60 \%$ de la mediana de los ingresos de la población en España en el momento de su estudio; esto es, $\mathbf{6 2 2 , 5} € /$ mes en 12 mensualidades para un adulto.. Para los menores, optan en su trabajo inicial por una cuantía equivalente al $20 \%$ de esa cantidad, lo que equivale a $1.494 € /$ año para los menores de edad.

Gimeno (2014 y ss.) opta por comparar con determinadas cuantías que sirven de referencia (tabla 1).

TABLA 1: CUANTÍAS DE REFERENCIA (2010)

\begin{tabular}{|l|c|c|}
\hline SUPUESTO & Anual & Mensual \\
\hline Salario mínimo & $8.866,20$ & 738,85 \\
\hline IPREM & $6.390,13$ & 532,51 \\
\hline Mínimo personal en el IRPF & $5.151,00$ & 429,25 \\
\hline Subsidio asistencial de desempleo & $5.112,10$ & 426,01 \\
\hline Rentas mínimas (media simple CCAA) & $5.792,5$ & 413,75 \\
\hline Pensión mínima no contributiva & $4.755,80$ & 396,32 \\
\hline Mínimo familiar en el IRPF (2+2) & $14.178,00$ & $1.181,50$ \\
\hline Pensión mínima con cónyuge a cargo & $7.697,20$ & 641,43 \\
\hline Renta básica mínima propuesta & $4.755,80$ & 396,32 \\
\hline Idem (2 adultos) & $9.511,60$ & 792,63 \\
\hline Idem (2+2) & $12.365,08$ & $1.030,42$ \\
\hline
\end{tabular}

Fuente: Gimeno (2014)

A partir de esos datos, opta por la opción más conservadora, fijando la cuantía en el equivalente a la pensión mínima no contributiva, es decir, al importe de las principales prestaciones asistenciales que han de ser absorbidas. Su propuesta, por tanto, ascendía en 2014 a 396,32 €/mes (4.755,80 €/año) para un adulto. En cambio, optaba por destinar a los menores un $30 \%$ de esa cifra $(1.426,74 € /$ año y menor) con la doble preocupación de las altas cifras de pobreza infantil y del problema de las familias monomarentales. Como puede verse, la cuantía de la renta para los menores es muy similar en ambos supuestos.

Conviene recordar que la renta básica tiene carácter de derecho individual, frente a la mayoría de las prestaciones asistenciales existentes que tiene una perspectiva predominantemente familiar. Por ello, son ilustrativas las dos últimas filas de la Tabla 1, que muestran cómo la cifra aparentemente modesta de la hipótesis Gimeno, asciende a una renta anual de 9.511, $60 €$ para una pareja, y a 12.365,08 € i, además, existen dos menores en el mismo núcleo familiar.

Podríamos decir, simplificadamente, que en la opción de Arcarons et al. la prioridad es erradicar la pobreza y en Gimeno es conseguir un modelo sostenible y más fácilmente financiable para favorecer su implantación. 
Para nuestras hipótesis, nos acercamos más a esta segunda preocupación, conscientes de que no conseguiríamos erradicar la pobreza pero sí reducirla sustancialmente, especialmente en los casos de menores en riesgo de exclusión social. Pensamos que esta opción es realista y sostenible, disminuye los posibles incentivos no deseados derivados de una renta incondicionada y consigue resultados muy razonables en la lucha contra la pobreza.

Como es sabido, se tramitó en el Congreso de los Diputados español la Ley de Iniciativa Legislativa Popular para establecer una Prestación de Ingresos Mínimos en el ámbito de protección de la Seguridad Social. La iniciativa, promovida por los sindicatos CCOO y UGT, se presentó el 19 de julio de 2016. La prestación equivale al $80 \%$ del IPREM (indicador público de renta de efectos múltiples), es decir, a 426 euros al mes en el momento de presentarse. Se percibe de forma indefinida mientras se cumplan los requisitos de acceso y se mantenga la situación de carencia de ingresos.

Aunque se configura como prestación no contributiva de último recurso, complementaria del sistema vigente de sostenimiento de rentas, nos sirve como referencia para la cuantía, que puede aceptarse como razonable para una primera implantación.

\section{TABLA 2: CUANTÍAS DE REFERENCIA (2019)}

\begin{tabular}{|l|c|c|}
\hline Dato & Mensual & "2+2" \\
\hline Línea de pobreza & $€ 684,00$ & $€ 1.436,50$ \\
\hline Salario mínimo & $€ 900,00$ & \\
\hline IPREM (indicador público de renta de efectos múltiples) & $€ 537,84$ & \\
\hline Renta básica (adulto) & $€ 430,27$ & \multirow{2}{*}{$€ 1.118,70$} \\
\hline Menores (30\%) & $€ 129,08$ & \\
\hline
\end{tabular}

Elaboración propia

Se parte, por tanto, de una renta básica equivalente al 80\% del IPREM, que supone en el momento actual $430,27 €$ /mes por adulto y de 129,08€ por menor. Obsérvese (tabla 2), que para una familia de dos personas adultas y dos menores, la renta básica se acerca al $80 \%$ de la línea de pobreza. Por lo tanto, la cuantía es razonablemente satisfactoria para el objetivo irrenunciable de erradicar la pobreza.

Conociéndose la cuantía de la renta básica, puede realizarse una primera estimación de su coste bruto global que resumimos en la tabla 3. 


\begin{tabular}{|c|c|c|c|}
\hline Modelo y año de referencia & Población & $N^{\circ}$ de personas & Coste (millones €/año) \\
\hline \multirow{3}{*}{$\begin{array}{l}\text { Arcarons et al. } \\
\text { (sin Comunidades Forales) (2010) }\end{array}$} & Menores de 18 años & 7.819 .887 & $11.682,9$ \\
\hline & Población adulta & 35.926 .543 & $268.371,0$ \\
\hline & TOTAL & 43.746 .430 & $280.054,0$ \\
\hline \multirow{3}{*}{$\begin{array}{l}\text { Gimeno } \\
\text { (con Comunidades Forales) (2010) }\end{array}$} & Menores & 14.181.051 & $20.232,7$ \\
\hline & Población adulta & 30.022 .368 & $142.780,4$ \\
\hline & TOTAL & 44.203 .419 & $163.013,1$ \\
\hline \multirow{3}{*}{$\begin{array}{l}\text { Actualización (Díaz) (con } \\
\text { Comunidades Forales) (2016) }\end{array}$} & Menores de 18 años & 8.307 .360 & $12.867,77$ \\
\hline & Población adulta & 38.249 .648 & $197.492,11$ \\
\hline & TOTAL & 46.557 .008 & $210.359,88$ \\
\hline
\end{tabular}

Elaboración propia

Las diferencias entre las estimaciones de población se explican por las distintas metodologías e hipótesis de cálculo. La primera se resalta en la propia tabla: en el primer caso, se excluyen de antemano las poblaciones de las comunidades forales: el sistema de concierto no se corresponde con el modelo vigente en el resto de España y la información tributaria correspondiente no está incluida en el panel de hogares del Instituto de Estudios Fiscales que se utiliza como fuente de estimaciones). En las otras dos están incluidas. El dato es relevante y deberá considerarse según los datos que se utilicen en las estimaciones posteriores.

En segundo lugar, los dos primeros estudios excluyen a los extranjeros sin residencia permanente, mientras que tal exclusión no se realiza en el trabajo de Díaz. Se opta por no reconocer automáticamente la renta básica a la población extranjera sin que se exija algún tipo de requisito previo, como los inherentes a la residencia permanente.

Llama la atención de forma especial la notoria diferencia de la cifra de "menores" en el estudio de Gimeno respecto a los otros dos. La explicación abre ya una de las primeras cuestiones para el debate. Gimeno considera que los menores deben solamente dejar de serlo, a los efectos del derecho a percibir la renta básica, cuando se emancipan. Al margen del debate adicional sobre qué ha de considerarse emancipación habilitadora del derecho, la razón de aplicar ese criterio es el temor a que se cree un desincentivo perverso en personas que ni estudian ni trabajan, que puedan conformarse por muchos años con esa cómoda situación de gastos pagados y renta automática incondicionada.

Las diferencias respecto al coste, además, han de entenderse relacionadas con las distintas cuantías aplicadas en cada caso. Recuérdese que la cifra elegida por Arcarons et al. es notoriamente superior a la utilizada en las otras dos estimaciones.

Como es obvio, las necesidades variarán según las decisiones normativas que se tomen respecto a cuantías y beneficiarios. De acuerdo con las hipótesis mencionadas, si elegimos la cifra del $80 \%$ del IPREM como adecuada para la implantación inicial de una renta básica en España, situaríamos las necesidades brutas de financiación entre los 175 y los 210 mil millones de euros, es decir, entre el 15,8\% y el 18,8\% del PIB. 
Es normal que estas cifras resulten llamativas y que la reacción inicial sea considerar que un programa de renta básica es inasumible. Pero, naturalmente, la renta básica implicaría la absorción de buen número de prestaciones o beneficios existentes, por lo que el coste neto de la medida sería notoriamente inferior.

Pasemos ahora a analizar esas figuras absorbibles y a estimar el ahorro consecuente en la financiación de la renta básica en España.

\section{AHORRO EN FIGURAS ABSORBIBLES}

Al tratarse la renta básica de una renta universal, y proporcionar un suelo para las familias, muchos de los actuales subsidios y ayudas del Estado quedarían absorbidos, o al menos, disminuidos en cierta proporción por dicha renta básica.

Así, las figuras absorbibles cuya cuantía fuera inferior o igual a la renta básica mensual, quedarían anuladas por la renta básica. De igual forma, cualquier ayuda cuya cuantía estuviese por encima de la renta básica mensual, quedaría disminuida en un importe igual al de dicha renta básica, de manera que, un perceptor de una ayuda cuya cuantía estuviese por encima de la renta básica, seguiría cobrando lo mismo que sin la renta básica.

Las cifras de dicho ahorro excluirán a las Comunidades Forales de País Vasco y Navarra, que no entran en estos cálculos de renta básica: su régimen foral propio las deja fuera de los datos fiscales disponibles para el resto de España. Esto no altera, sin embargo, la validez global de los resultados que se obtienen.

Dentro de las figuras absorbibles, algunas son claras e indiscutibles: todas las prestaciones asistenciales, subsidios y ayudas familiares, ayudas por exclusión social, etc. serían sustituidas por una figura única que toma en consideración directamente esos factores y los atiende, según se reconoce, de forma más eficiente.

Menos unanimidad despiertan, como ahora veremos, las prestaciones contributivas, ya sean pensiones, ya sea el subsidio de desempleo.

Los modelos que estamos revisando incluyen algunos supuestos que no siempre coinciden en unos y otros y que llevan implícitos supuestos de menos evidente relación con la renta básica que los anteriores. 


\begin{tabular}{|c|c|c|c|}
\hline \multirow{2}{*}{\multicolumn{2}{|c|}{ TABLA 4 - RESUMEN DEL TOTAL DE PRESTACIONES }} & \multicolumn{2}{|c|}{ TOTAL 2010} \\
\hline & & Beneficiarios & Euros al año \\
\hline \multirow{2}{*}{ Pensiones contributivas } & Pensiones contributivas & 6.279 .845 & 75.172 .609 .670 \\
\hline & Complemento a mínimos & 2.391 .173 & 5.935 .143 .166 \\
\hline \multirow{4}{*}{ Prestaciones no contributivas } & $\begin{array}{l}\text { Pensiones no contributivas de la } \\
\text { seguridad social }\end{array}$ & 453.295 & 2.170.277.693 \\
\hline & $\begin{array}{l}\text { Complemento de pensión anual por } \\
\text { alquiler de vivienda }\end{array}$ & 14.210 & 7.503 .175 \\
\hline & Pensiones asistenciales & 16.189 & 33.965 .170 \\
\hline & Prestaciones por discapacidad & 36.977 & 49.847 .241 \\
\hline \multirow{4}{*}{ Prestaciones por desempleo } & Nivel contributivo & 1.471 .826 & 22.684 .498 .000 \\
\hline & Nivel asistencial - Subsidio & 1.291 .467 & 7.424.103.000 \\
\hline & $\begin{array}{l}\text { Subsidio trab. eventuales agrarios } \\
\text { (REAS) }\end{array}$ & 153.761 & 800.189 .000 \\
\hline & Renta activa de inserción & 125.680 & 636.114 .000 \\
\hline \multirow{2}{*}{ Prestaciones por hijos a cargo } & Periódicas & 791.980 & 1.207 .987 .560 \\
\hline & Pago único & 1.331 .482 & 1.955.699.240 \\
\hline \multirow{2}{*}{ Prestaciones de maternidad } & Prestaciones de maternidad & 326.752 & 1.820 .510 .798 \\
\hline & Prestaciones de paternidad & 275.637 & 225.543 .356 \\
\hline \multirow{2}{*}{ Prestaciones autonómicas } & Renta mínima de inserción & 511.295 & 766.731 .832 \\
\hline & Ayudas de emergencia social & 406.603 & 161.256.103 \\
\hline Prestaciones locales & $\begin{array}{l}\text { Prestaciones básicas de servicios } \\
\text { sociales }\end{array}$ & 8.047 .920 & 831.527 .750 \\
\hline \multirow[b]{2}{*}{$\begin{array}{l}\text { Gasto en atención a la } \\
\text { dependencia }\end{array}$} & $\begin{array}{l}\text { Créditos para la Atención a la } \\
\text { Dependencia }\end{array}$ & \multirow[b]{2}{*}{8.000 .009} & 1.840.177.000 \\
\hline & $\begin{array}{l}\text { Créditos suplementos gasto cuotas } \\
\text { seguridad social de los cuidados } \\
\text { familiares }\end{array}$ & & 331.930 .000 \\
\hline \multicolumn{2}{|l|}{ Fuente: Gimeno (2014) } & TOTAL 2010 & 124.055.613.755 \\
\hline
\end{tabular}

Puede servir de primera aproximación la tabla 4, en la que Gimeno distinguía entre los distintos tipos de prestaciones. Las marcadas en fondo gris serían parte del primer grupo citado; las contributivas del segundo y las restantes del tercero. Aunque dentro de este último aparecerán conceptos adicionales.

Veamos cómo contempla cada modelo estas prestaciones.

Para Gimeno, la absorción que supone la renta básica de las prestaciones dispersas preexistentes (las sombreadas en la tabla 4) supone ya una suma de financiación estimada en 21.239,17 millones de euros. 
Para las prestaciones contributivas adopta la presunción (como hacen también Arcarons et al. y que debatiremos más adelante) de que se absorbe la cuantía equivalente a la renta básica (quedando lo que exceda como componente variable). Ello supone una cifra estimada en 29.865,9 millones de euros en pensiones y de 6.999,71 en subsidio de desempleo, un total conjunto de $36.865,4$ millones.

Un tercer bloque de ahorros lo liga Gimeno a la eliminación en el IRPF de los mínimos personales y familiares, la supresión del beneficio para las aportaciones a los planes de pensiones (una desgravación ligada a la base imponible que resulta fuertemente regresiva) y las prestaciones familiares. Todo lo cual podría suponer, de acuerdo con el panel del IRPF del IEF para 2010, una cifra adicional de 27.583,24 millones de euros.

La simplificación que supone un sistema de renta básica frente a la vigente proliferación de programas podría suponer una reducción importante de recursos administrativos que hoy se dedican a la gestión y control de las prestaciones absorbidas. No resulta fácil estimar la cuantía de esa renta, más siendo conscientes de la rigidez de la burocracia administrativa. No obstante, opta por incorporar el mismo criterio seguido por Arcarons et al., y calcula el ahorro en el $50 \%$ del gasto administrativo asociado a los gastos sociales según la estadística SEPROSS de Eurostat: 2.532,55 millones de euros adicionales.

Por último, Gimeno añade que la redistribución que implica la implantación de la renta básica provocará un estímulo al consumo, con el consiguiente efecto multiplicador sobre la demanda agregada y, por ende, sobre la recaudación tributaria. Estima que se recuperaría por esta vía un 4\% del total empleado en el programa, $6.520,52$ millones de euros.

En resumen, Gimeno considera que de forma automática se recuperan 94.740,88 millones de euros, algo más del $60 \%$ del coste bruto antes calculado.

Veamos ahora el mismo proceso en Arcarons et al., así como la actualización realizada por Díaz (recordemos que proponiendo una cuantía de renta básica menor) siguiendo, en lo posible, la misma metodología, y con algunos retoques que iremos comentando.

Las figuras absorbibles identificadas por ambas aproximaciones son: pensiones, clases pasivas del Estado, prestaciones por desempleo, subsidios y ayudas a la vivienda, subsidios y ayuda a la familia e hijos, ayudas por exclusión social, becas, reservistas sin destino (FFAA y FCSE), 50\% de los gastos administrativos, sacerdotes y reclusos.

Los campos marcados por el asterisco son aquellos en los que Díaz introduce algunos ajustes:

- Es bastante común que viudos cobren tanto pensión de viudedad, como su pensión habitual, y que huérfanos cobren becas y pensiones de orfandad, por lo que corrige los casos en los que se les deduce dos veces a viudos o huérfanos la cuantía de la renta básica mensual.

- También Díaz ajusta los subsidios a la vivienda, pues prefiere no incluirlos por mezclarse las ayudas de la vivienda con la situación patrimonial. 
La siguiente tabla muestra la comparación en el ahorro de los dos modelos que estamos ahora analizando:

\begin{tabular}{|c|c|c|}
\hline \multicolumn{3}{|c|}{ TABLA 5: IMPORTE DE FIGURAS ABSORBIDAS (Millones de euros) } \\
\hline PRESTACIÓN & Arcarons et al. & Actualización Díaz \\
\hline Subsidios y ayudas a la familia & $3.661,68$ & $3.230,43$ \\
\hline Subsidios y ayudas a la vivienda & $2.164,76$ & $2.482,23$ \\
\hline Ayudas por exclusión social & $1.957,84$ & $2.482,23$ \\
\hline Pensiones & $54.023,56$ & $31.210,31$ \\
\hline Prestaciones por desempleo & $21.405,84$ & $18.208,90$ \\
\hline Becas & $1.917,07$ & $1.919,92$ \\
\hline Clases pasivas del Estado & $3.815,71$ & $2.974,65$ \\
\hline Reservistas sin destino & 258,95 & 176,20 \\
\hline Sacerdotes & 126,50 & 87,22 \\
\hline Reclusos & 533,57 & 295,44 \\
\hline \multirow[t]{2}{*}{$50 \%$ gastos administrativos } & $2.356,81$ & $2.377,73$ \\
\hline & \multicolumn{2}{|c|}{ AJUSTES } \\
\hline Subsidios y ayudas a la vivienda & - & $-2.482,23$ \\
\hline Orfandad & - & $-493,15$ \\
\hline Viudedad & - & $-9.511,13$ \\
\hline TOTAL & $92.222,29$ & $52.958,75$ \\
\hline
\end{tabular}

Elaboración propia

Como es lógico, la menor cuantía de la renta básica en el segundo modelo implica ahorros menores en prestaciones absorbidas que en la aproximación inicial de Arcarons et. al. En el Anexo se detallan las fuentes e hipótesis aplicadas por Díaz para realizar sus estimaciones.

\section{COSTE Y FINANCIACIÓN DE LA RENTA BÁSICA}

El repaso a las estimaciones de necesidades de financiación de cara a la implantación de una renta básica nos aporta un panorama con algunas luces pero con muchas sombras.

Por un lado, hemos visto conceptos tomados en consideración que necesitarían un debate sosegado sobre las razones a favor y en contra de su absorción por parte de la nueva figura o/y sobre la forma en que habría de implementarse. En un epígrafe siguiente repasaremos algunos de ellos. 
Por otro lado, se observan diferencias importantes en las estimaciones. Es obvio que algunas de las hipótesis de partida son diferentes y condicionan los resultados. Pero hay casos en los que se aplican prácticamente las mismas hipótesis y se toman teóricamente las mismas referencias, pero los resultados divergen. Utilizar una fuente estadística u otra, incluso consultarlas en distintas fechas puede significar partir de datos diferentes.

Por ello, hay que tomar todas las estimaciones como aproximaciones necesitadas de un estudio más detallado y profundo. Deberían ser las propias administraciones del Estado las que lideraran ese esfuerzo.

\begin{tabular}{|l|c|c|c|}
\hline \multicolumn{1}{|l|}{ TABLA 6: NECESIDAD DE FINANCIACIÓN (Millones de euros) } \\
\hline 1. Coste bruto de la renta básica & GIMENO & ARCARONS ET AL. & DÍAZ \\
\hline 2. Prestaciones absorbidas & 163.013 & 280.054 & 197.738 \\
\hline (\% de 2 sobre 1) & $85.687,81$ & $92.222,29$ & $52.958,75$ \\
\hline 3. Administración & $52,57 \%$ & $32,93 \%$ & $26,78 \%$ \\
\hline 4. Incremento recaudación & $2.532,55$ & $2.356,81$ & $2.377,73$ \\
\hline 5. "Autofinanciación" (2+3+4) & $6.520,52$ & & - \\
\hline 6. “Déficit" (1-5) & $94.740,88$ & $94.579,10$ & $55.336,48$ \\
\hline \% "déficit"/total (directo) & $68.272,17$ & $185.474,90$ & $140.798,53$ \\
\hline \% "déficit"/total (homogéneo) & $41,88 \%$ & $66,23 \%$ & $72,02 \%$ \\
\hline
\end{tabular}

Elaboración propia

Como puede verse en la tabla 6, que resume las estimaciones relatadas, las necesidades netas de financiación varían notoriamente entre los distintos trabajos: desde la previsión más optimista -la de Gimeno que estima una autofinanciación superior al 50\% de las necesidades brutas- al "pesimismo" de Díaz, según cuyas previsiones las prestaciones absorbidas cubren poco más de la cuarta parte del gasto bruto estimado. Es decir, aparentemente, Díaz estima una necesidad de financiación doble a la que calcula Gimeno.

Arcarons et al., lógicamente, muestran también una alta necesidad de financiación, dos tercios del gasto total, en buena medida por partir de una cuantía más elevada de la prestación.

Como hemos visto, una diferencia importante es que Gimeno incorpora los ahorros en el IRPF directamente a la estimación del importe de las figuras absorbidas mientras que en los otros dos modelos esas cifras (bastante coincidentes en cuanto a los conceptos) se incluyen al estimar la propuesta de estimación del IRPF necesario. Si aplicáramos a los otros dos modelos el ahorro equivalente, el porcentaje de "déficit" sobre el total bajaría al $56,89 \%$ en el caso de Arcarons et al, al $58,80 \%$ en el de Díaz.

Si también consideramos la recuperación recaudatoria estimada por Gimeno a través del incremento en el consumo y el efecto multiplicador consiguiente, los porcentajes descienden al 52,89\% y al 55, 50\%. Las estimaciones ya no son tan dispares.

Tomando como razonable el valor medio de los tres trabajos, podemos concluir que el $50 \%$ del coste de implantación de una renta básica se autofinancia con las figuras absorbidas y efectos colaterales. O, lo que es lo mismo, que aquellos porcentajes del PIB que tanto asustaban de principio, ya han quedado reducidos a la mitad. 
En todo caso, es evidente que la implantación de una renta básica universal no puede financiarse exclusivamente con las figuras absorbidas, sea cual sea la cuantía aceptada y sean cuales sean los supuestos que se incorporen. ¿Cómo podemos cubrir esa diferencia para hacer viable financieramente la reforma?

Las propuestas de los dos modelos de financiación iniciales (Arcarons et al. y Gimeno), son notoriamente diferentes, quizás también por las diferentes necesidades estimadas. Los primeros proponen una reforma radical del IRPF que permita recaudar lo necesario para llegar al objetivo marcado; el segundo busca una solución más continuista con el actual sistema impositivo, que prácticamente no se toca (con las matizaciones que veremos), y para recuperar el "exceso" de gasto, arbitra una "devolución" parcial de la renta básica percibida, de forma progresiva, a través del propio IRPF. Repasemos ambas propuestas.

Díaz procede a aplicar el modelo de Arcarons et al. con los datos actualizados que hemos visto. Las hipótesis de trabajo de las que parten ambos trabajos para financiar la renta básica son, pues, comunes:

- Integración de la base del ahorro en la base general.

- Eliminación de compensación entre rendimientos del trabajo y del capital.

- Eliminación de mínimos personales o familiares, reducciones de la base por planes de pensiones u otras razones, y de deducciones de la cuota (vivienda, incentivos, etc.).

- Financiación mediante un tipo único de IRPF tanto para rendimientos del trabajo como del capital.

A partir de esas hipótesis, se procede a comparar el cálculo del coste objetivo de financiación para la renta básica de los dos modelos con la base imponible fiscal de rendimientos del trabajo y del capital proporcionadas por la Agencia Tributaria para los años 2010, y 2016, lo que nos sirve para el cálculo del tipo único final aplicable, suficiente para financiar la renta básica.

\begin{tabular}{|l|c|c|}
\hline \multicolumn{1}{|l|}{ TABLA 7: CÁLCULO DEL TIPO FIJO DEL IRPF } & Arcarons et al. & Díaz \\
\hline Coste Renta Básica & $280.054 \mathrm{M}$ & $197.738 \mathrm{M}$ \\
\hline Ahorro en Figuras Absorbibles & $-92.222,29 \mathrm{M}$ & $-52.958,75 \mathrm{M}$ \\
\hline Actual recaudación del IRPF & $67.807,53 \mathrm{M}$ & $69.949 \mathrm{M}$ \\
\hline COSTE OBJETIVO & $285.044,53 \mathrm{M}$ & $214.728 \mathrm{M}$ \\
\hline TIPO ÚNICO & $49 \%$ & $37,13 \%$ \\
\hline Cuantía de la Renta Básica & $622,50 € / \mathrm{mes}$ & $430,27 € / \mathrm{mes}$ \\
\hline
\end{tabular}

Elaboración propia

A partir del coste total bruto de la renta básica, deducimos los ahorros en las figuras absorbibles y sumamos la recaudación del IRPF ${ }^{3}$. Se obtiene así el coste objetivo que debemos financiar. Solo queda estimar el tipo único aplicable necesario para alcanzar esa cifra objetivo. La tabla 7 nos ofrece el resultado en ambas estimaciones.

\footnotetext{
${ }^{3}$ Hay que subrayar que en el caso del modelo de Arcarons et al., el tipo único de IRPF ha sido calculado mediante los datos de un microsimulador, mientras que, en el modelo de Díaz, el cálculo de IRPF se estima directamente a partir de la cifra de Base Imponible de rendimientos del trabajo y del capital de 2016, cifrado por la Agencia Tributaria en 578.266 millones de $€$.
} 
La idea de un tipo único resulta chocante para muchas personas. Conviene recordar que la renta básica juega doblemente: como renta igual para todos (porcentaje más relevante cuanto menor el ingreso previo), y como mínimo exento. Ello dota ya al impuesto de una importante progresividad. Precisamente la menor cuantía de la renta básica supone también un menor mínimo exento y, por tanto, propicia una mayor recaudación con un tipo menor, como sucede en el modelo Díaz. Por otra parte, la forma de cálculo realizada por este último modelo no incorpora las minoraciones de renta que han acompañado a la implantación de la renta básica, por lo que probablemente sobrevalora la recaudación.

Como muestran las estimaciones de Arcarons et al., el cambio global (renta básica y nuevo IRPF), resulta claramente progresivo, favoreciendo al setenta por ciento de la población y perjudicando de forma creciente a los hogares de los tres deciles de mayores ingresos.

Gimeno opta por otro modelo más conservador. Considera que implantar una renta básica encuentra de entrada fuertes resistencias sociales y políticas. Es cierto que esas resistencias van decreciendo, pero siguen siendo un obstáculo difícil de salvar. Por ello, no es evidente que los representantes políticos, incluso los que podrían ser proclives a la idea, se lancen a defenderla abiertamente ante una opinión pública inicialmente reacia. Si a esa situación añadimos el debate sobre un cambio radical del IRPF, incluyendo además la poco intuitiva idea del tipo único, las dificultades políticas se multiplican.

Un tipo fijo implica un crecimiento mayor del tipo medio efectivo en los primeros escalones de renta, aplanándose la curva correspondiente ${ }^{4}$. Ello supone una presión fiscal elevada para niveles de renta media/ media-alta, que quizás tampoco es deseable. Es obvio que una tarifa más suavemente progresiva tiene un coste recaudatorio importante y que, por ello, resulta explicable la opción utilizada. Pero implica un factor político adicional en contra de la reforma.

Frente a las resistencias detectadas, es muy reducida la oposición ciudadana a una renta mínima garantizada para quienes lo necesiten. De ahí que Gimeno proponga una renta básica estructurada como renta mínima automática e incondicionada. El derecho se reconoce de entrada como universal. Pero si la realidad muestra que los ingresos de unas personas son elevados, la renta mínima resulta innecesaria y procede por tanto que el Estado la "recupere". Basta para ello retocar la tarifa actual del impuesto (y las retenciones correspondientes).

${ }^{4}$ Ejemplo de evolución del tipo medio para un tipo fijo del 49\%, base medida en miles de euros y mínimo exento de $20.000 €$.

Tipo medio con tipo fijo

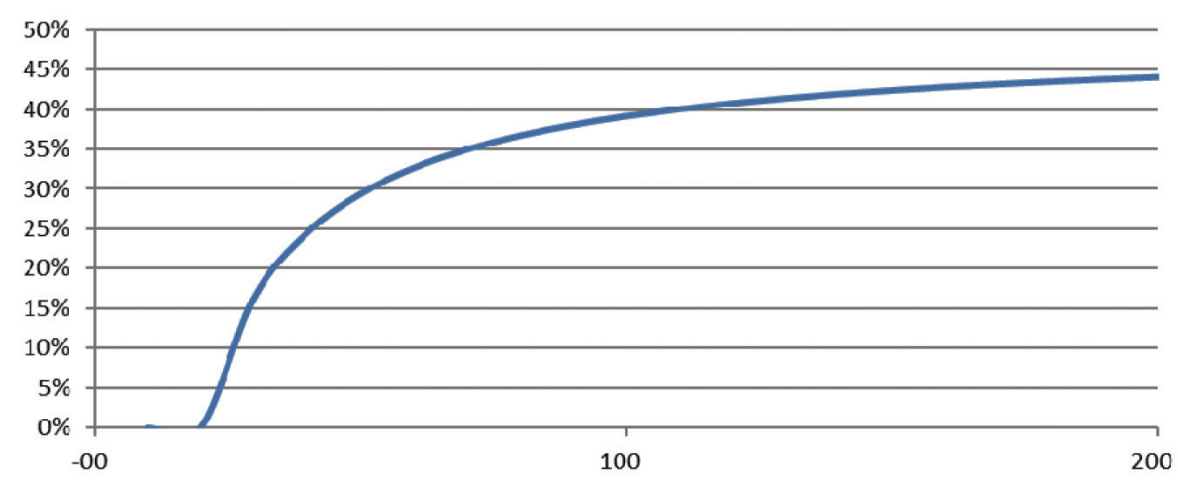


Es necesario recuperar ese 42\% (en su previsión "optimista", 50\% tomando la media de las estimaciones) del coste del programa de renta básica, necesario para que cuadren las cuentas y se garantice la sostenibilidad del programa. Para ello, propone Gimeno incorporar un mecanismo de recuperación (a modo de recargo en el impuesto) con las características siguientes:

- Debe garantizar la totalidad del ingreso básico a los primeros deciles de renta. Podría empezar a recuperarse a partir del cuarto decil de renta, de forma progresiva, alcanzando la totalidad para los dos últimos. Así, la renta básica jugaría de forma completa o casi completa para cerca de la mitad de la población y de forma muy relevante para más de dos tercios de la población.

- El recargo debe subir de forma gradual de forma que se evite cualquier error de salto.

- La neutralidad podría aconsejar que el máximo de recargo fuera equivalente a la renta básica efectivamente recibida, es decir, inferior o como máximo igual al equivalente a su devolución total, según el nivel de renta. No obstante, para conseguir recuperar el $40 \%$ objetivo, sería necesario admitir un techo superior si no queremos dañar la progresividad del beneficio.

- La parte que rebasara a la estricta equivalencia con lo recibido debiera evitar que se penalizara en función del número de hijos. Por ello, quizás debería fijarse en función exclusivamente del número de adultos.

- Por último, sería conveniente que el recargo afectara fundamentalmente a los niveles superiores de renta, y de forma especial a las rentas diferentes del trabajo, por ser estas las más claramente penalizadas en nuestro actual IRPF.

La tabla 8 muestra un sencillo ejemplo gráfico de la propuesta. En este caso, se ha supuesto que las personas de las tres primeras decilas cobran íntegramente su renta básica y que a partir de la siguiente comienza a recuperarse de forma progresiva a través de las retenciones del IRPF (línea de puntos). De esta forma, la renta básica se cobraría integra por quienes más lo necesitan y no recibirían nada las personas de las decilas superiores de renta. En este ejemplo, el coste neto de la renta básica se reduce a la mitad y, por tanto, conforme a las estimaciones analizadas, resulta financieramente viable y netamente progresiva.

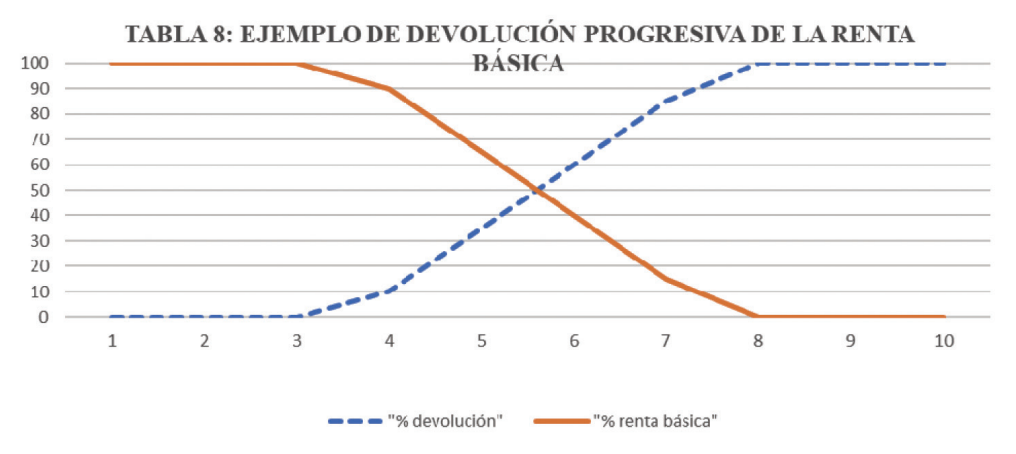

Esta segunda opción reduce algunos de los obstáculos señalados para la alternativa de Arcarons et al. No es menos cierto que buena parte de su virtualidad está ligada a la estimación de cuál es el "déficit" de la renta básica una vez descontados los ingresos por figuras absorbidas y otras causas. Un porcentaje del entorno del cincuenta por ciento, como el que parece deducirse de las estimaciones comparadas, permite esta financiación casi neutral en términos absolutos. Porcentajes más elevados exigirían necesariamente una reforma tributaria y un debate más amplio sobre las prioridades sociales y su peso relativo.

Y todo ello, sin perjuicio de algunos debates que planteamos más adelante. 


\section{LA RECIENTE PROPUESTA DE ARCARONS, RAVENTÓS Y TORRENS}

Como se hacía notar más arriba, con posterioridad a la realización de los cálculos anteriores, los tres autores citados han publicado (Noviembre de 2018) una nueva propuesta más elaborada y compleja, referida a datos de 2015. Aunque la muestra se refiere solo a la ciudad de Barcelona, la riqueza de datos de disponibles hace la estimación de resultados extremadamente relevante.

Se ofrecen 12 variantes o escenarios:

- Cuantía de la renta básica:

» 7.800 euros anuales, 650 mensuales en 12 pagas.

" 8.400 euros anuales $(700 € /$ mes $)$

" 9.802 euros anuales ( $816,83 € /$ mes)

- Tarifa del IRPF

" Tipo único

" Tipos progresivos por tramos

- Financiación complementaria:

" Sí.

»No.

Ya en este esquema destacan dos novedades: se abren dos hipótesis adicionales como la tarifa progresiva y la financiación adicional por vía distinta al IRPF.

La tarifa progresiva presentaría tipos marginales que oscilan, según las hipótesis, entre el 29,46\% y el 64\% (este último solo para las rentas por encima de los 90.000 euros, y en la opción de umbral de renta mínima de 9.802 euros y sin financiación complementaria).

Los posibles ingresos adicionales se estiman en 500 millones de euros que podrán provenir, se propone, de un impuesto sobre la riqueza del $1 \%$, aplicado al $5 \%$ superior más rico.

Más importante que estas dos innovaciones es el hecho de que la cuantía individual de la renta básica se calcule para el hogar completo, aplicando la conocida escala de equivalencia modificada de la OCDE ( 1 para el primer adulto mayor de 18 años, 0,5 para los restantes con edad superior a los 14 años y 0,3 para los menores de esta edad). La cantidad resultante se repartiría equitativamente entre sus integrantes de forma individualizada.

Esta novedad es muy importante: mejora la equidad en las prestaciones recibidas por cada hogar en función de sus necesidades y reduce algo el coste global de la propuesta, pero introduce un factor de mayor complejidad administrativa.

Otro dato relevante y digno de resaltarse es que, además de la redistribución esperada de los niveles altos de renta a los más bajos, habría una redistribución de 431 millones de euros de hombres a mujeres, lo que equivale a un $1,4 \%$ de la renta bruta total de la ciudad de Barcelona (que, recordemos, es la población analizada). Se trata de un buen dato en favor de la corrección de la brecha de ingresos entre hombres y mujeres. 


\section{ALGUNOS DEBATES PENDIENTES}

Los cuadros de financiación enunciados han ido incluyendo algunos supuestos no exentos de debate. Algunas de las partidas que se han asumido como absorbidas es probable que no lo sean fácilmente.

Por ejemplo, Arcarons y Díaz incluyen a sacerdotes, reservistas sin destino y reclusos como perceptores de prestaciones que quedarían absorbidas por la renta básica. Los argumentos son admisibles: están recibiendo una prestación pública que puede equipararse a otras prestaciones asistenciales cuya absorción no se discute. La renta básica sería redundante. Pero no es menos cierto que, por unas u otras razones, surgirán resistencias para esa absorción. No representan ni el $1 \%$ de las cifras que estamos necesitando, por lo que tampoco merece la pena detenerse mucho en ello.

Hay prestaciones incluidas en las estimaciones que son autonómicas, por rentas mínimas o de inclusión (o denominaciones semejantes) u otras ayudas de emergencia social. Si la renta básica se concibe como una competencia estatal gestionada por el sistema de la Seguridad Social, resulta problemático asumir que se lograrán aportar para su financiación todos los recursos actualmente gestionados desde las Comunidades Autónomas.

Hay otras consideraciones que podrían apoyar la idea de que no es deseable la absorción. Por un lado, es obvio que la renta básica no soluciona todos los problemas de necesidad: hay algún colectivo que queda sería (inmigrantes no residentes, por ejemplo), hay supuestos excepcionales, hay problemas de vivienda adicionales, hay necesidades especiales... Corresponde precisamente a las Comunidades Autónomas completar todas esas necesidades complementarias con políticas asistenciales más cercanas a la ciudadanía.

Por otro lado, también es evidente que las necesidades de supervivencia no son las mismas en unos lugares que en otros, en entornos urbanos que en entornos rurales... La implantación de una renta básica universal a nivel estatal sería así una magnífica oportunidad para la revisión de las actuales rentas mínimas autonómicas por políticas más selectivas y ajustadas a necesidades.

Tanto por estas razones como por el principio general de no perjudicar a las prestaciones vigentes del estado de bienestar, nos inclinamos por no considerar absorbibles las prestaciones por dependencia ni los gastos de servicios sociales en general.

Pero el debate más relevante, en cuanto a absorción de figuras, se refiere a las prestaciones contributivas (v., por ejemplo, De la Fuente, 2015).

En el caso de las pensiones, se argumenta, en primer lugar, que la renta que se percibe es una contraprestación debida a cambio de las cotizaciones realizadas a lo largo de la vida activa, que son un salario diferido o socializado, que se va acumulando para el periodo posterior a la jubilación. Absorber el importe de la renta básica sería, desde esta perspectiva, una cierta expropiación injusta.

Este argumento tiene mucho de falaz. Sabemos que el sistema de pensiones no es, ni por aproximación, un sistema de capitalización. De hecho, la longevidad actual implica que la gran mayoría de los pensionistas recibe más de lo que cotizó. Por lo tanto, incluso aceptando el argumento, no habría injusticia alguna si la pensión se divide en dos mitades, la una equivalente a la renta básica y la otra ligada a las cotizaciones.

La confusión aparece por ligar rígidamente el sistema de pensiones a las cotizaciones que las financian. ¿Qué ocurriría si el sistema tributario fuere otro y no existieran las cotizaciones como ahora las conocemos, sino otras figuras impositivas generales que financiaran la seguridad social de la misma forma que financian las restantes prestaciones públicas? La presunta equivalencia se derrumba. 
Por otra parte, se argumenta que si la renta básica absorbe la parte equivalente de las pensiones, beneficiaría a toda la población menos a los jubilados. Recordemos que la introducción de la renta básica beneficia a las personas situadas en los niveles bajos de ingresos pero perjudica a los demás altos ingresos. Lo mismo ocurriría con los pensionistas: los que estén percibiendo pensiones extremadamente bajas verían mejorar su prestación. Y solo los muy ricos (previsiblemente perceptores de ingresos adicionales a las pensiones públicas) saldrían perjudicados.

En todo caso, así como los ingresos involucrados en los primeros conceptos discutidos y discutibles son relativamente pequeños y marginales, aquí estamos ante una cuestión crucial. Si la renta básica se añadiera a las pensiones contributivas de forma automática, las cuentas dejarían de casar. Las pensiones contributivas absorbidas representan el componente fundamental de ahorro ante la introducción de la renta básica.

Por lo tanto, existen solo dos opciones:

1.Adaptar el sistema de pensiones sobre la base de dos componentes: una parte fija, igual para todas las personas (la renta básica), que absorbería las pensiones asistenciales de todo tipo (incluidos los complementos a mínimos) y la parte equivalente de cuantas pensiones fueran superiores en cuantía a ese mínimo. Y una parte variable complementaria, ligada a las cotizaciones realizadas por cada persona. La cuantía presupuestaria de la suma de ambas partes debería resultar de partida, semejante a la actualmente destinada al pago de pensiones.

2.Introducir la renta básica solamente para ciudadanos menores de 65 años (o la edad de jubilación que corresponda), garantizando una pensión mínima equivalente a la renta básica, tanto asistencial como contributiva.

Los resultados financieros de ambas opciones no serían muy diferentes, coherentes con las estimaciones realizadas. Solo con microdatos podríamos estimar el resultado redistributivo resultante de una u otra opción, aunque, probablemente, tampoco diferirán mucho en términos globales.

En el caso del subsidio de desempleo, los cálculos realizados son perfectamente asumibles en una perspectiva estática, es decir en el momento de la implantación. Pero se plantean algunos problemas en una perspectiva dinámica.

El subsidio de desempleo nació en un entorno de pleno empleo, para sostener las rentas de quienes pierden temporalmente su empleo y hasta tanto encuentren uno nuevo. La idea es que durante unos meses, la persona afectada pueda mantener unos ingresos no demasiado alejados de los previos a su nueva situación. Si se absorbiera íntegramente por la renta básica, la caída de ingresos sería muy importante para quien entra en el paro.

Deberíamos arbitrar aquí también dos partes en el subsidio de desempleo: una fija, equivalente a la renta básica, y otra variable que completara el ingreso en función de lo cotizado y su salario en el último puesto de trabajo. Esta parte tendería a reducirse con el tiempo.

No es difícil de articular pero, si queremos mantener el mismo objetivo de sostenimiento de rentas vigente, probablemente el ahorro en subsidio de desempleo sería algo menor al que estimaban los dos primeros trabajos estudiados. 
Por lo tanto, se subraya que existen diversos aspectos relevantes que debatir, si bien no se considera que (salvo en el caso de las pensiones contributivas) se alteren de forma significativa las conclusiones alcanzadas sobre la financiación de esta política pública.

Más transcendente es el último punto que queremos destacar. En todos los casos se ha partido, no podía ser de otra forma, de los datos de IRPF y de financiación a través de esa figura impositiva.

Un problema que no puede ignorarse es la estructura profundamente desequilibrada de nuestro actual IRPF, en el que las rentas de trabajo soportan casi en exclusiva su recaudación. En efecto, el peso de las rentas de trabajo en la recaudación del impuesto prácticamente duplica al que significan en el conjunto de la renta nacional.

Ello se debe tanto al trato de favor que reciben otras rentas -especialmente las del capital financiero- como al diferente control al que son sometidos los asalariados por cuenta ajena con respecto a la mayoría de las otras fuentes de renta.

Por ello, conviene ver con especial cuidado cualquier relación con el impuesto para no perpetuar o agravar sus injusticias.

Las propuestas de Arcarons et al. y de Díaz corrigen en buena medida esa pega puesto que contemplan en su reforma un IRPF que trata por igual las rentas de trabajo que las de capital.

No así la propuesta de Gimeno que, de entrada, mantiene invariada la estructura del impuesto. Por ello, será especialmente necesario corregir esa propuesta si quiere evitarse la inequidad inherente al impuesto vigente. La solución propuesta supondría, de hecho, un perjuicio especial para la clase media y media alta que vive de su salario.

La propuesta de Gimeno podía, de entrada, considerarse más viable, realista y posibilista. Pero es deseable que ese $50 \%$ que falta se financie, al menos en parte, por vías ajenas al IRPF. Sería, por tanto, mucho más deseable un esquema parecido al que se refleja en la tabla 9. En ese ejemplo, el beneficio completo alcanza a los cuatro primeros deciles y todas las personas reciben al menos parte, incluso en la última decila, como mínimo exento perfectamente legítimo.

Tabla 9: Ejemplo de devolución progresiva parcial de la renta básica

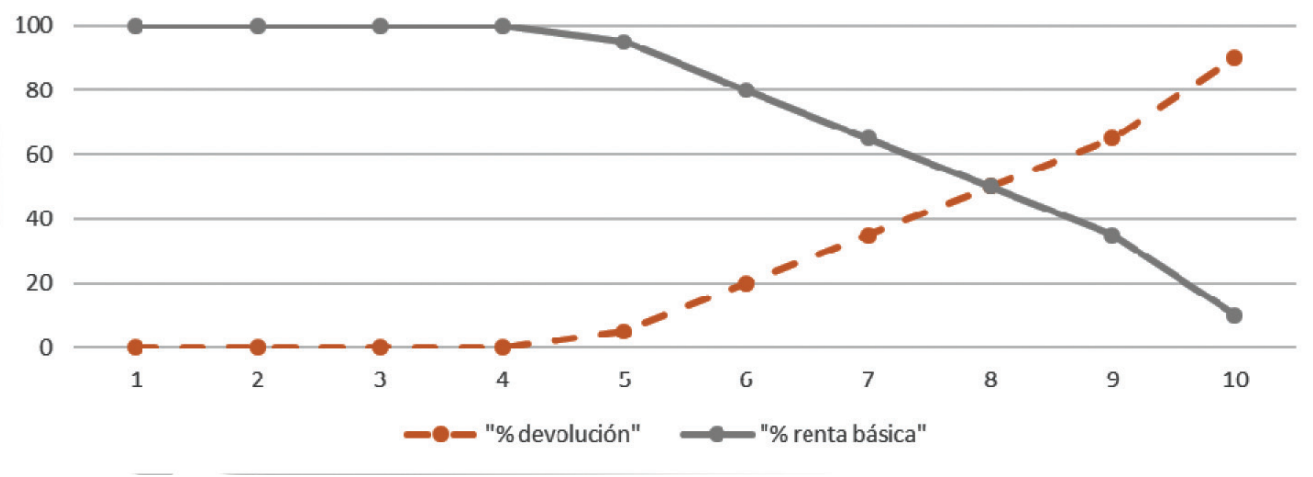


De esa forma, más de la mitad de las necesidades de financiación estimadas se recupera automáticamente y solo necesitamos algún ingreso adicional para el 20-25\% del coste bruto estimado.

Son muchas las vías posibles para ello. Sin duda, la primera debe ir en línea con la propuesta de Arcarons et al. y Díaz: acercar al máximo el tratamiento que reciben las rentas de capital (y buena parte de las empresariales) al soportado por las de trabajo. Es preciso también combatir el privilegio de quienes utilizan las guaridas fiscales.. Este soporta un conjunto de tributos de los que, de nuevo, se libra el financiero. Por ejemplo, la propiedad de un inmueble supone una imputación de renta sujeta a tributación cuando no hay ingreso por alquiler; sin embargo, la tenencia de activos financieros no supone pago alguno. Primar unos destinos del ahorro frente a otros, amén de ineficiente, tiene habitualmente consecuencias regresivas.

En general, los ingresos adicionales que necesita nuestro sistema tributario (recordemos, siete puntos del PIB de presión fiscal por debajo de la media europea) han de provenir de suprimir el conjunto de privilegios que disfrutan actualmente determinadas rentas y colectivos, sin justificación defendible.

Hay que suprimir buena parte de las exenciones y bonificaciones fiscales, que merman la recaudación impositiva y responden mayoritariamente a presiones de sectores específicos, las más de las veces con resultados regresivos, en beneficio de unos pocos, con difícil explicación desde la perspectiva del interés general. De hecho, algunas supresiones en el IRPF se han incorporado a las estimaciones recaudatorias, según hemos visto.

Lo anterior es especialmente aplicable al Impuesto de sociedades, donde el conjunto de beneficios supone que el tipo medio sea ridículamente bajo, con un tratamiento inequitativo adicional en favor de las grandes corporaciones (con más medios para evadir legalmente sus cargas tributarias) y en contra de la pequeña y mediana empresa.

Es preciso suprimir el privilegio del que gozan las transacciones financieras. Cualquier transmisión patrimonial soporta un tributo, del que se exime a las financieras. El impuesto sobre este tipo de transacciones se convierte así, amén de otras ventajas, en una elemental exigencia de justicia.

Es preciso suprimir el privilegio de quienes gozan de una posición dominante desproporcionada por el mero hecho de nacer en una determinada familia y lugar. El impuesto sobre las grandes herencias y donaciones es un requisito insoslayable de redistribución. Se confunde a la población anunciando un presunto beneficio para las clases medias. El impuesto debe contemplar un suficiente mínimo exento para las transmisiones de padres a hijos, de forma que un alto porcentaje de la población quede prácticamente fuera de la obligación de pagar o soporte cifras mínimas. Sorprende, por cierto, que muchos de quienes defienden la supresión de este impuesto se opongan a la renta básica de ciudadanía con el argumento de que estimula la vagancia. Parece que cuatrocientos y pico euros al mes llevan a la vagancia, pero millones de euros no.

Es preciso combatir el privilegio del que gozan las grandes empresas tecnológicas que evitan pagar impuesto alguno gracias a su ubicación "en la nube". Las denominadas GAFAs (por Google, Apple, Facebook, Amazon), pero también muchas otras multinacionales que localizan artificialmente sus beneficios donde la presión tributaria sea mínima. 
Es preciso combatir el privilegio de quienes, en general, utilizan cualquier forma de fraude y evasión fiscal. Casi las tres cuartas partes del fraude fiscal corresponden a las grandes empresas y a los grandes patrimonios; el $17 \%$ a las PYMES; el $9 \%$ a los autónomos y tan sólo un $2 \%$ al sector no empresarial, según las estimaciones de GESTHA (Sindicato de Técnicos del Ministerio de Hacienda). Está claro de dónde proviene buena parte de la injusticia de nuestro sistema fiscal y dónde deben situarse las prioridades de actuación de la Agencia Tributaria. Es preciso también, combatir el privilegio de quienes utilizan las guaridas fiscales.

A todo lo anterior podemos sumar la imposición medioambiental, con un amplio recorrido por delante y ese doble dividendo que lleva aparejada: recaudatorio y corrector de excesos contaminantes.

En suma: si parecen claras las ventajas de implantación de una renta básica como política eficiente de sostenimiento de rentas y lucha contra la pobreza, el necesario debate de cómo financiarla encuentra modelos que muestran su viabilidad financiera. 


\section{ANEXO: ESTIMACIONES DE DÍAZ DE FIGURAS ABSORBIDAS}

Se detalla en este anexo la procedencia de cada una de las cifras estimadas de gastos absorbidos por la renta básica, en la actualización del modelo Arcarons et al. realizada por Díaz (2019), Se especifican tanto las fuentes utilizadas, como los cálculos aplicados para cada figura absorbible, incluyendo, en su caso, enlaces públicos a dicha información.

\section{- Ahorro en pensiones:}

Dicho ahorro se calcula directamente de la página de la Seguridad Social ESTADISS, que nos ofrece la información tanto por tramos de cuantía, como por Comunidades Autónomas, por lo que nos es muy sencillo excluir a País Vasco y Navarra.

La tabla siguiente refleja los cálculos realizados:

\begin{tabular}{|c|c|c|c|c|}
\hline TRAMOS DE CUANTÍA & TOTAL PENSIONES & PROMEDIO TRAMO & COSTE ANUAL & AHORROS A 430,27€ \\
\hline Hasta 150,00 euros & 112.091 & 100 & 1400 & $156.927 .400,00 €$ \\
\hline De 150,01 a 250,00 & 173.969 & 200 & 2800 & $487.113 .200,00 €$ \\
\hline De 250,01 a 300,00 & 109.015 & 275 & 3850 & $419.707 .750,00 €$ \\
\hline De 300,01 a 350,00 & 182.956 & 325 & 4550 & $832.449 .800,00 €$ \\
\hline De 350,01 a 400,00 & 129.443 & 375 & 5250 & $668.345 .275,32 €$ \\
\hline De 400,01 a 450,00 & 194.464 & 425 & 5950 & $1.004 .064 .303,36 €$ \\
\hline De 450,01 a 500,00 & 154.514 & 475 & 6650 & $797.792 .865,36 €$ \\
\hline De 500,01 a 550,00 & 125.323 & 525 & 7350 & $647.072 .726,52 €$ \\
\hline De 550,01 a 600,00 & 175.430 & 575 & 8050 & $905.787 .193,20 €$ \\
\hline De 600,01 a 650,00 & 635.554 & 625 & 8750 & $3.281 .517 .834,96 €$ \\
\hline De 650,01 a 735,89 & 1.005 .748 & 693 & 9702 & $5.192 .918 .303,52 €$ \\
\hline De 735,90 a 800,00 & 266.473 & 768 & 10752 & $1.375 .864 .052,52 €$ \\
\hline De 800,01 a 900,00 & 520.225 & 850 & 11900 & $2.686 .046 .529,00 €$ \\
\hline De 900,01 a $1.000,00$ & 349.008 & 950 & 13300 & 1.802.012.065,92€ \\
\hline De $1.000,01$ a $1.100,00$ & 325.551 & 1.050 & 14700 & $1.680 .897 .945,24 €$ \\
\hline De $1.100,01$ a $1.200,00$ & 276.843 & 1.150 & 16100 & $1.429 .406 .851,32 €$ \\
\hline De $1.200,01$ a $1.300,00$ & 248.316 & 1.250 & 17500 & $1.282 .115 .103,84 €$ \\
\hline De $1.300,01$ a $1.400,00$ & 218.639 & 1.350 & 18900 & $1.128 .885 .630,36 €$ \\
\hline De $1.400,01$ a $1.500,00$ & 213.153 & 1.450 & 20300 & $1.100 .560 .095,72 €$ \\
\hline De $1.500,01$ a $1.600,00$ & 185.176 & 1.550 & 21700 & $956.108 .130,24 €$ \\
\hline De $1.600,01$ a $1.700,00$ & 161.409 & 1.650 & 23100 & $833.393 .405,16 €$ \\
\hline De $1.700,01$ a $1.800,00$ & 130.417 & 1.750 & 24500 & $673.374 .271,08 €$ \\
\hline De $1.800,01$ a $1.900,00$ & 112.522 & 1.850 & 25900 & $580.978 .091,28 €$ \\
\hline De $1.900,01$ a $2.000,00$ & 107.883 & 1.950 & 27300 & $557.025 .820,92 €$ \\
\hline De $2.000,01$ a $2.100,00$ & 99.417 & 2.050 & 28700 & $513.313 .831,08 €$ \\
\hline De $2.100,01$ a $2.200,00$ & 98.000 & 2.150 & 30100 & $505.997 .520,00 €$ \\
\hline De $2.200,01$ a $2.300,00$ & 89.493 & 2.250 & 31500 & $462.073 .837,32 €$ \\
\hline De $2.300,01$ a $2.400,00$ & 75.358 & 2.350 & 32900 & $389.091 .439,92 €$ \\
\hline De $2.400,01$ a $2.500,00$ & 82.466 & 2.450 & 34300 & $425.791 .749,84 €$ \\
\hline De $2.500,01$ a $2.600,00$ & 97.198 & 2.550 & 35700 & $501.856 .601,52 €$ \\
\hline De $2.600,01$ a $2.614,94$ & 12.271 & 2.607 & 36498 & $63.358 .118,04 €$ \\
\hline De $2.614,95$ a $2.614,97$ & 235.211 & 2.615 & 36610 & $1.214 .450 .843,64 €$ \\
\hline Más de $2.614,97$ & 40.144 & 2.650 & 37100 & 207.273.106,56€ \\
\hline TOTAL CON FORALES & 6.943 .680 & & & $34.763 .571 .692,76 €$ \\
\hline SOLO FORALES & & & & 3.553.259.156,16€ \\
\hline \multicolumn{2}{|c|}{ TOTAL SIN FORALES } & & & $31.210 .312 .536,60 €$ \\
\hline
\end{tabular}

Las cifras se pueden encontrar en el siguiente enlace: http:/www.seg-social.es/wps/portal/wss/internet/ EstadisticasPresupuestosEstudios/Estadisticas/EST23/EST24/EST192 


\section{- Ahorro en clases pasivas del Estado}

Aquí se incluyen los 619.486 funcionarios adscritos al programa MUFACE, MUGEJU o ISFAS, en cuanto que funcionarios, personal de justicia y Fuerzas Armadas, respectivamente. Se aplica un ahorro total equivalente anual de 5.163,23 €/año al número de beneficiarios, resultando el ahorro total equivalente:

"Pensionistas equivalentes: 619.486.

" Ahorro total: $3.198,55$ millones de euros

La fuente utilizada para la obtención del número de pensionistas equivalentes de estos tres colectivos se encuentra en

http://www.clasespasivas.sepg.pap.hacienda.gob.es/sitios/clasespasivas/es-ES/QuienesSomos/ EstadisticasInformes/Documents/N\%C3\%BAmero\%20de\%20pensiones\%20abonadas\%20por\%20 Clases\%20Pasivas\%20Junio\%202016.pdf.

\section{- Ahorro en prestaciones por desempleo:}

En este caso la estimación se ha realizado a partir de la cifra estimada por Arcarons el al. para el primer estudio, que obtenía un ahorro de 26.347,6 millones de $€$, para una cuantía anual de renta básica de 7.471€ anuales. Díaz estima la cuantía del ahorro para 2016 mediante una simple proporción con la cuantía de 5.163

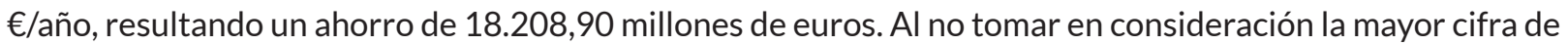
parados en 2016, la estimación ha de considerarse inferior al ahorro real y, por tanto, más conservadora respecto al ahorro efectivo de haberse utilizado las cifras de ese año.

" Ahorro en subsidios para vivienda, familia e hijos, y exclusión social

Estas tres cifras se obtienen directamente de la estadística ESSPROS, publicada en 2017 por el CES (Consejo Económico y Social) (http://www.ces.es/documents/10180/4509980/Inf0117.pdf)

De dicho documento se extraen los ahorros en subsidios y ayudas para la vivienda, familia e hijos, y por exclusión social siguientes:

- Ayuda y subsidios a la vivienda: 2.482,23 millones de euros.

- Ayuda y subsidios a familia e hijos: 3.230,43 millones de euros.

- Ayuda y subsidios por exclusión social: 2.482,23 millones de euros.

\section{- Ahorro en becas:}

Las becas se dividen en dos: las que proporciona la Administración general del estado a través del Ministerio de Educación, y las proporcionadas por cada Comunidad Autónoma (excluyendo de nuevo País Vasco y Navarra).

La información de la cuantía sobre dichas becas está disponible en el Ministerio de Educación, Cultura y Deporte en los siguientes enlaces:

" http://estadisticas.mecd.gob.es/EducaJaxiPx/Datos.htm?path=/Recursosecon/Becas/2015-16/ TodasEnse//I0/\&file=Todas2.px\&type=pcaxis.

" http://estadisticas.mecd.gob.es/EducaJaxiPx/Tabla.htm?path=/Recursosecon/Becas/2015-16/ TodasEnse//IO/\&file=Todas1.px\&type=pcaxis\&L=0. 
Las cifras aportadas son las siguientes, separadas en dos tablas, una para las becas del Ministerio, y otra para las becas de las Administraciones Educativas de las CCAA:

\begin{tabular}{|l|r|}
\hline & \multicolumn{2}{|c|}{ Ministerio } \\
\hline TOTAL & \\
\hline Andalucía & 920.355 \\
\hline Aragón & 218.423 \\
\hline Asturias, Principado de & 18.583 \\
\hline Balears, Illes & 14.297 \\
\hline Canarias & 10.779 \\
\hline Cantabria & 45.136 \\
\hline Castilla y León & 8.593 \\
\hline Castilla-La Mancha & 57.321 \\
\hline Cataluña & 33.451 \\
\hline Comunitat Valenciana & 120.697 \\
\hline Extremadura & 105.054 \\
\hline Galicia & 25.705 \\
\hline Madrid, Comunidad de & 50.339 \\
\hline Murcia, Región de & 122.364 \\
\hline Rioja, La & 36.631 \\
\hline Ceuta & 4.415 \\
\hline Melilla & 8.033 \\
\hline Sin especificar C.A. & 8.226 \\
\hline UNED & 309 \\
\hline & 19.179 \\
\hline
\end{tabular}

\begin{tabular}{|l|r|}
\hline TOTAL & $\begin{array}{r}\text { Admones. educativas } \\
\text { de las CC.AA. }\end{array}$ \\
\hline Andalucía & 999.560 \\
\hline Aragón & 258.993 \\
\hline Asturias, Principado de & 27.541 \\
\hline Balears, Illes & 5.452 \\
\hline Canarias & 11 \\
\hline Cantabria & 74.367 \\
\hline Castilla y León & 9.997 \\
\hline Castilla-La Mancha & 55.376 \\
\hline Cataluña & 8.293 \\
\hline Comunitat Valenciana & 3.474 \\
\hline Extremadura & 128.086 \\
\hline Galicia & 2.934 \\
\hline Madrid, Comunidad de & 114.142 \\
\hline Murcia, Región de & 118.472 \\
\hline Rioja, La & 39.115 \\
\hline Ceuta & 11.212 \\
\hline Melilla & 0 \\
\hline Sin especificar C.A & 0 \\
\hline UNED & 47 \\
\hline & 261 \\
\hline
\end{tabular}

En total, entre las dos suman 1.919,92 millones de euros. Para el estudio de Arcarons se procedió de forma análoga y, de hecho, la cifra es prácticamente idéntica (1.917 millones de euros).

\section{- Ahorro en reservistas sin destino:}

Para este apartado, se ha tomado como referencia el número de reservistas sin destino en España, multiplicado por un factor de 0,93 (para excluir a las Comunidades Forales), y multiplicado por 5.163,23 $€$ del importe anual de la renta básica en el estudio de Díaz. Como fuentes se han empleados las siguientes páginas de internet:

https://publicaciones.defensa.gob.es/anuario-estadistico-militar-ano-2016.html, desde donde se puede destacar el Anuario Estadístico Militar de las Fuerzas Armadas del Estado, donde aparece el número de reservistas, con el que se procede a realizar los cálculos:

\begin{tabular}{lrr} 
& No Beneficiarios & Ahorro a $5163,23 €$ \\
FFAA & 14799 & $76.410 .640,77 €$ \\
Guardia Civil & 7603 & $39.256 .037,69 €$ \\
CNP & 14292 & $73.792 .883,16 €$ \\
Total con forales & & $189.459 .561,62 €$ \\
\hline \multicolumn{1}{l}{ TOTAL SIN FORALES } & & $176.197 .392,31 €$
\end{tabular}




\section{- Ahorro en sacerdotes, reclusos:}

En ambos supuestos se aplica la hipótesis de coste cero en subsidio por estar percibiendo ya, los primeros, una transferencia del Estado, y, para los segundos, porque al cumplir condena en la cárcel, están privados de derechos civiles y tienen cubiertas sus necesidades básicas por el Estado. Las estadísticas que contienen el número de reclusos en España y de sacerdotes, se encuentran en los enlaces que siguen:

" http://www.ine.es/jaxi/Tabla.htm?path=/t38/p604/a2000/I0/\&file=0500001.px\&L=0.

" https://www.conferenciaepiscopal.es/datos-generales-la-iglesia-espana/.

Con las dos cifras de personas reclusas y sacerdotes, se pasa a calcular el ahorro a 5.163€/anuales, resultando una cifra de 317,67 millones de euros en el primer caso, de 93,78 millones en el segundo.

\section{- Ahorro en costes de la Administración Pública}

Tal como se ha señalado en el texto, se considera que se produce un ahorro en gastos en la Administración, por haber disminuido sustancialmente las necesidades burocráticas con un sistema casi automático como el de la renta básica.

Para calcular dicho ahorro, se toma el $50 \%$ del gasto administrativo total, en la página 47 , del informe publicado por el CES en 2017, con los datos de 2014:

http://www.ces.es/documents/10180/4509980/Inf0117.pdf.

Este $50 \%$ de 4.755,46 millones de euros, da una cifra de ahorro de: $2.377,73$ millones de euros, para el estudio de Díaz. Como se ha señalado, los tres estudios siguen el criterio inicialmente adoptado por Arcarons et al.

\section{- Disminución del ahorro por pensiones de viudedad y de orfandad:}

Como se ha dicho anteriormente, en el estudio de Díaz, se ha contabilizado el hecho de que viudos y huérfanos estén recibiendo dos pensiones simultáneamente, los primeros, por recibir su pensión habitual y la pensión de viudedad, y los segundos, por recibir becas de educación y pensión de orfandad a la vez. Con objeto de no computar dos veces el ahorro correspondiente, se ha sumado el coste que tienen dichas pensiones duplicadas de orfandad y viudedad anualmente.

Para la estimación se ha cogido el número de personas que perciben en España una pensión de viudedad y orfandad, y se ha multiplicado por $5.163 €$, que es la cuantía anual de la renta básica, que se ha restado en la tabla-resumen del ahorro en las figuras absorbibles. Las siguientes estadísticas permiten obtener el número de personas beneficiarias de pensiones de orfandad y viudedad en España:

» https://es.statista.com/estadisticas/607850/numero-de-personas-que-percibian-una-pension-porviudedad-por-tramo-espana/.

» https://es.statista.com/estadisticas/607880/personas-que-percibian-una-pension-por-orfandad-portramo-espana/.

De esta forma, se obtienen las cifras de 530,27 millones de euros para las prestaciones de orfandad y de 10.227,03 para viudedad. 


\section{REFERENCIAS BIBLIOGRÁFICAS}

- AGENCIA TRIBUTARIA (2016). Informe anual de recaudación tributaria. https://www.agenciatributaria. es/static_files/AEAT/Estudios/Estadisticas/Informes_Estadisticos/Informes_Anuales_de_Recaudacion_ Tributaria/Ejercicio_2016/IART16.pdf

- ARCARONS, J.; RAVENTÓS, D. y TORRENS, LL. (2013): “Un modelo de financiación de la Renta Básica técnicamente factible y políticamente no inerte". http://www.sinpermiso.info.ARCARONS, J., RAVENTÓS, D., y TORRENS, L. (2017). Renta básica incondicional. En Una propuesta de financiación racional y justa (pág. 168). Barcelona, España: Ediciones del Serbal.

- ARCARONS, J., RAVENTÓS, D., y TORRENS, L. (2018). Nuevo modelo de financiación de la Renta Básica. http://www.sinpermiso.info/textos/nuevo-modelo-de-financiacion-de-la-renta-basica

- ARCARONS, J.; DOMĖNECH, A.; RAVENTÓS, D. y TORRENS, LL. (2014): “Un modelo de financiación de la Renta Básica para el conjunto del Reino de España: sí, se puede y es racional". http://www.sinpermiso.info/ textos/index.php?id=7535.

- BASQUIAT, M. y BROCHÉ, g. (2015): Liber, un revenu de liberté pour tous : Une proposition d'impôt négatif en France. Éditions de l'ombre. Generation Libre. Paris.

- CONSEJO ECONÓMICO Y SOCIAL DE ESPAÑA (CES). (2017). Informe Políticas Públicas para combatir la pobreza en España. http://www.ces.es/documents/10180/4509980/Inf0117.pdf

- DE LA FUENTE, M. (2015): Renta Básica Universal y la Seguridad Social. VIENTO SUR. Número 138/ Febrero 2015. Pgs.102-109.

- DÍAZ OYARZÁBAL, J. (2019): Estudio de Renta Básica Universal en España, UPM, Madrid.

- EUROSTAT. (2018). ESSPROS. Expenditure: main Results: http://appsso.eurostat.ec.europa.eu/nui/ show.do?dataset=spr_exp_sum\&lang=enGIMENO, J.A. (2014): "Coste/beneficio de una renta básica". Presupuesto y gasto público, $n^{\circ} 76$, págs. 141-158.

- GIMENO, J.A. (2015). “Aproximación a una renta básica sostenible”. En Ayala, L. y Ruiz Huerta, J. (eds.): Segundo informe sobre la desigualdad en España. Fundación Alternativas, Madrid, págs. 97-103.

- INSTITUTO NACIONAL DE ESTADÍSTICA (2016). Boletín mensual de estadística: Diciembre 2016. http://www.ine.es/jaxi/Tabla.htm?path=/t38/p604/a2000/I0/\&file=0500001.px\&L=0

- MINISTERIO DE DEFENSA. Secretaría General Técnica. Unidad de Estadística del Órgano Central. (2016). Estadística de personal militar y de personal del cuerpo de la Guardia Civil. https://publicaciones.defensa.gob.es/anuario-estadistico-militar-ano-2016.html

- MINISTERIO DE EDUCACIÓN, CULTURA Y DEPORTE. Gobierno de España. (2016). Becas y ayudas al estudio: estadísticas. http://estadisticas.mecd.gob.es/EducaJaxiPx/Datos.htm?path=/Recursosecon/ Becas/2015-16/TodasEnse//I0/\&file=Todas2.px\&type=pcaxis

- MINISTERIO DE EDUCACIÓN, CULTURA Y DEPORTE. Gobierno de España. (2018). Recursos económicos. Gasto público del año 2016: https://www.mecd.gob.es/servicios-al-ciudadano-mecd/ estadisticas/educacion/recursos-economicos/gasto-publico/2016.html

- MINISTERIO DE HACIENDA Y ADMINISTRACIONES PÚBLICAS. Gobierno de España. (2016). Clases Pasivas: http://www.hacienda.gob.es/Boletines/2016/48245.pdf

- MINISTERIO DE HACIENDA. Gobierno de España. (2016). Coste de Personal y Pensiones Públicas: http://www.hacienda.gob.es/en-gb/CDI/Paginas/CostePersonalPensiones/CostePersonalPensiones. aspx 
- MINISTERIO DE HACIENDA. Gobierno de España. (2016). Pensiones por colectivos beneficiarios: los distintostiposdepensionesy prestaciones deClases pasivas.http://www.clasespasivas.sepg.pap.hacienda. gob.es/sitios/clasespasivas/es-ES/QuienesSomos/Estadisticas/nformes/Paginas/Beneficiarios2016.aspx

- RED RENTA BÁSICA (2016). Encuesta: 64\% de la población de Europa votarían a favor de la Renta básica universal. http://www.redrentabasica.org/rb/encuesta-64-de-la-poblacion-de-europa-votarian-a-favorde-la-renta-basica-universal/

- SEGURIDAD SOCIAL (2018). Pensiones por CCAA y provincias. Total Sistema. http://www.seg-social.es/ wps/portal/wss/internet/EstadisticasPresupuestosEstudios/Estadisticas/EST23/EST24/EST192

- STATISTA (2016). Número de personas beneficiarias de pensión de orfandad por tramos. https://es.statista. com/estadisticas/607880/personas-que-percibian-una-pension-por-orfandad-por-tramo-espana/

- STATISTA (2016). Número de personas beneficiarias de pensión de viudedad. https://es.statista.com/ estadisticas/607850/numero-de-personas-que-percibian-una-pension-por-viudedad-por-tramoespana/

- VAN PARIJS, P., Y VANDERBORGTH, Y. (2017). Ingreso básico. Una propuesta racional para una sociedad libre y una economía sensata. Grano de Sal. Ciudad de México, México. 